\title{
IDENTIFICAÇÃO DE FASES MINERAIS EM MICROESTRUTURAS DE PELOTAS ATRAVÉS DO EMPREGO DA TÉCNICA DE EBSD*
}

\author{
Marcos Meyer Machado ${ }^{1}$ \\ Nádia Alves Simão ${ }^{2}$ \\ Ana Paula Carcheno Souza Vianna ${ }^{3}$
}

\begin{abstract}
Resumo
A determinação de características microestruturais como base de controle de qualidade tem demonstrado cada vez mais a sua importância nos processos de aglomeração de minério de ferro. A linha de pesquisa do presente trabalho envolveu o desenvolvimento de técnicas de embutimento de pelotas seccionadas em resina condutora, de desbaste e polimento da superfície das amostras e emprego do microscópio eletrônico de varredura em conjunto com a técnica de Electron Backscattering Difraction. Por meio das técnicas empregadas de microscopia baseadas em parâmetros cristalográficos específicos, foram obtidas ilustrações forescatter e mapas de fases minerais ferríticas, silicatos e óxido de ferro que caracterizam a microestrutura de pelotas tratadas termicamente em forno piloto. $\mathrm{O}$ desenvolvimento desta metodologia promove subsídios para estabelecer futuramente uma relação das estruturas observadas em escala microscópica com as propriedades reconhecidas e mapeadas no produto final.
\end{abstract}

Palavras-chave: Pelotas; Fases minerais; EBSD

\section{MINERAL PHASES IDENTIFICATION ON MICROSTROCTURE OF IRON OXIDE PELLET BY ELECTRON BACKSCATTERING DIFRACTION TECHNIQUE}

\section{Abstract}

The determination of microstructural characteristics as quality control has been increasingly employed in iron ore agglomeration processes. This research involved the techniques development of inlay in conductive resin, buffing and polishing samples surfaces and the Electron Backscattering Diffraction technique application. Through the combined techniques of microscopy, maps and images forescatter were obtained containing iron oxide, ferrites and silicates phases illustrating the microstructure of iron oxide pellets based on their different crystallographic parameters.

Keywords: Pellet; Mineral phases; EBSD

Engenheiro metalurgista, Unidade Técnica, DIPE, Vale, Vitória, ES, Brasil.

Técnico de laboratório de microscopia, Engenharia de Processo, DIPE, Vitória, ES, Brasil.

Técnico de laboratório de microscopia, Engenharia de Processo, DIPE, Vitória, ES, Brasil. 


\section{INTRODUÇÃO}

As pelotas diferem do minério granulado por diversas razões atreladas as propriedades físicas e metalúrgicas às quais são pré-determinadas e definidas para a aplicação no processo siderúrgico posterior. Essas propriedades dependem diretamente das características mineralógicas e morfológicas do minério de ferro, da adição de aglomerantes, fluxantes (calcário), combustível sólido (antracito), além de parâmetros de tratamento térmico como temperatura e tempo [5]. Os componentes químicos adicinados através dos insumos (cálcio, magnésio e sílica) em conjunto com a matriz ferritica (hematita e magnetita) além de gases redutores gerados pela combustão do antracito, reagem entre si durante o tratamento térmico formando diferentes fases microestruturais às quais podem ser relacionadas com a obtenção das propriedades do produto [1;2].

\subsection{Reações envolvidas no processo de tratamento térmico de pelotas}

O processo de tratamento térmico inicia-se por um pré-aquecimento das pelotas secas a uma temperatura próxima de $900{ }^{\circ} \mathrm{C}$ de forma a preparar termicamente $\mathrm{O}$ leito para exposição de uma atmosfera de temperaturas elevadas. Nesta outra etapa, as pelotas são submetidas à máxima temperatura do ciclo térmico, que atinge valores da ordem de 1250 a $1350^{\circ} \mathrm{C}$, dependendo do tipo de minério e da composição química da pelota que está sendo produzida [3]. Sob estas condições ocorre a consolidação final do produto, através da sinterização das partículas de minério de ferro e das reações de escorificação envolvendo componentes de ganga ácida e básica [4].

O calor envolvido no processo de queima potencializa as reações químicas envolvendo os constituintes contidos na pelota (minério, calcário, bentonita, cal, carvão, etc). Essas reações são fundamentais para promover o endurecimento das pelotas e consequentemente as propriedades físicas e metalúrgicas. Nesta etapa de processo, algumas reações iniciam-se e desencadeiam outras segundo condições únicas e até pontuais. Podemos citar as principais reações envolvidas [5]:

$\rightarrow$ Reações de combustão do carvão (iniciam-se a $500^{\circ} \mathrm{C}$ ).

- $\mathrm{C}+1 / 2 \mathrm{O}_{2} \rightarrow \mathrm{CO}$

(Equação 1)

- $\mathrm{C}+\mathrm{O}_{2} \rightarrow \mathrm{CO}_{2}$

$\rightarrow$ Reações de decomposição dos hidratos (iniciam-se a $550^{\circ} \mathrm{C}$ ).

- $\mathrm{CaCO}_{3} \rightarrow \mathrm{CaO}+\mathrm{CO}_{2}$

(Equação 3)

- $\mathrm{MgCO}_{3} \rightarrow \mathrm{MgO}+\mathrm{CO}_{2}$

(Equação 4)

$\rightarrow$ Reações de escorificação com formações de novas fases (iniciam-se a $900^{\circ} \mathrm{C}$ )

- $\mathrm{CaO}+\mathrm{Fe}_{2} \mathrm{O}_{3} \rightarrow \mathrm{CaO} . \mathrm{Fe}_{2} \mathrm{O}_{3}$

(Equação 5)

- $\mathrm{MgO}+\mathrm{Fe}_{2} \mathrm{O}_{3} \rightarrow \mathrm{MgO}$. $\mathrm{Fe}_{2} \mathrm{O}_{3}$

(Equação 6)

- $2 \mathrm{MgO}+\mathrm{SiO}_{2} \rightarrow 2 \mathrm{MgO} . \mathrm{SiO}_{2}$

(Equação 7)

- $2 \mathrm{CaO}+\mathrm{SiO}_{2} \rightarrow 2 \mathrm{CaO} . \mathrm{SiO}_{2} \ldots \ldots$. Silicatos

(Equação 8)

- $3 \mathrm{SiO}_{2}+2 \mathrm{Fe}_{3} \mathrm{O}_{4}+2 \mathrm{CO} \rightarrow 3\left(2 \mathrm{FeO} . \mathrm{SiO}_{2}\right)+\mathrm{CO}_{2} \ldots .$. Fayalita (Equação 9)

$\rightarrow$ Reação de oxidação da magnetita (de $200^{\circ} \mathrm{C}$ a $1000{ }^{\circ} \mathrm{C}$ [Jiang et al. 2008]

- $2 \mathrm{Fe}_{3} \mathrm{O}_{4}+1 / 2 \mathrm{O}_{2} \rightarrow 3 \mathrm{Fe}_{2} \mathrm{O}_{3}+\mathrm{dQ}$ (geração de calor)

(Equação 10) 


\section{2 - MEV - Microscópio Eletrônico de Varredura e a técnica EBSD - Electron Backscatter Diffraction}

O (MEV) é um dos instrumentos mais versáteis disponíveis para exames e análises morfológicas e caracterizações químicas composicionais. Imagens são produzidas à medida que um feixe de elétrons varre a amostra e o sinal recebido por um detector de elétrons é mostrado em um monitor formando imagens em três dimensões. Além do mais, tamanha versatilidade pode ser ampliada quando se acoplam novos detectores, como por exemplo, a difração de elétrons retro-espalhados.

A partir da década de 90, quando se tornaram comercialmente disponíveis, os sistemas automatizados MEV-EBSD tiveram grande aceitação em laboratórios de metalurgia, ciência dos materiais e geofísica, devido à simplicidade operacional e à rápida aquisição de dados.

A técnica de difração de elétrons retroespalhados (EBSD) lança mão da operabilidade desse equipamento em diversas escalas para fornecer informações microestruturais quantitativas sobre a natureza cristalográfica dos metais, minerais, semicondutores e materiais cerâmicos [6].

\subsubsection{Princípio de funcionamento do sistema de EBSD}

O objetivo dessa técnica consiste em adquirir e analisar os Padrões de Kikuchi característicos do retículo cristalino de um material, retornando diversas informações. Para tanto, a amostra é posicionada com sua superfície inclinada em um ângulo de $70^{\circ} \mathrm{com}$ o eixo $X$ do microscópio - ou $20^{\circ} \mathrm{com}$ o feixe de elétrons sendo essa a situação de maior difração do feixe incidente nos planos cristalográficos do material, de acordo com a Lei de Bragg [7; 8] (Figura 1).

Dessa forma, assim como as técnicas convencionais de MEV e microscopia óptica, mapas de EBSD podem ser usados para visualizar a microestrutura do material e também acessar informações sobre tamanho e forma dos grãos em duas e três dimensões. A partir desses mesmos dados de orientação de todo o retículo cristalino do material, pode ser gerada uma enorme variedade de informações visuais e analíticas que incluem orientação cristalina preferencial ou textura, distribuição de fases, estado de deformações e variações locais de deformação residual, além de distribuição e energia dos contornos de grão [9]. 
A - Montagem da amostra no Sistema MEV-EBSD

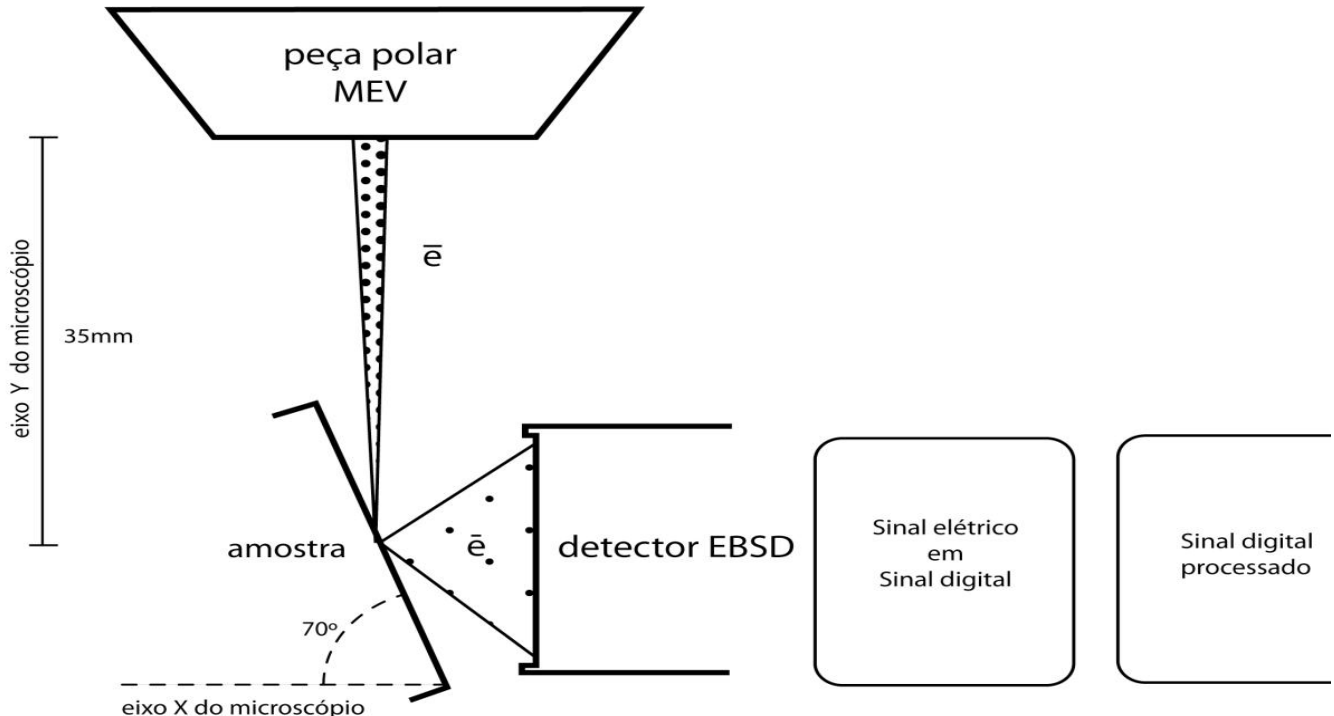

B - Leitura pontual de orientação cristalográfica no EBSD

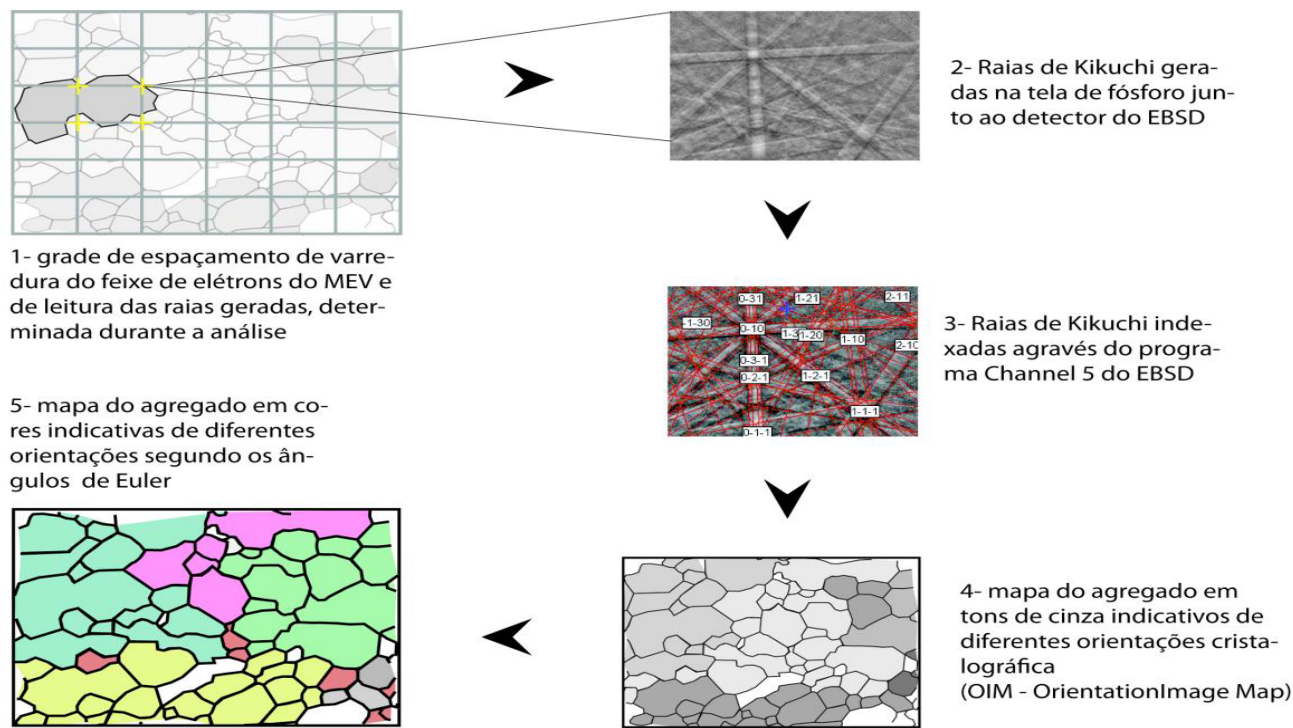

Figura 1. A) Esquema de funcionamento do EBSD - detecção e processamento; B) EBSPs Indexação, análise, e formação dos mapas de orientação. [Adaptado de Barbosa, 2009]

\section{MATERIAIS E MÉTODOS}

Com base nas especificações químicas do produto Vale para redução direta, amostras de pelotas foram preparadas e tratadas termicamente em usina piloto com perfis de temperatura diferentes. Amostras de $180 \mathrm{~kg}$ foram alocadas para seleção e análise microscópica.

\subsection{Determinação da composição química das pelotas}

O teor de Fe foi determinado pelo método a úmido, em que o Fe é titulado com uma solução de dicromato de potássio $0,1 \mathrm{~N}$. O teor de $\mathrm{SiO}_{2}, \mathrm{Al}_{2} \mathrm{O}_{3}, \mathrm{P}, \mathrm{Mn}, \mathrm{CaO}, \mathrm{MgO}$ e $\mathrm{TiO}_{2}$ foram determinados por fluorescência de raios-X - SIMULTIX 14 de RIGAKU CORPORATION $®$. A perda por calcinação (PPC) foi realizada em um forno Mufla a $1000^{\circ} \mathrm{C}$ - QUIMIS. 


\subsection{Embutimento e preparação de amostras}

As amostras de pelotas foram selecionadas inicialmente a partir dos resultados do teste de compressão a frio. De posse do resultado médio, foram coletados cacos gerados com valores próximos a média (desvio de $\pm 10 \mathrm{DaN} / \mathrm{pel}$ ) e a $100 \mathrm{DaN} / \mathrm{pel}$ abaixo. As amostras selecionadas foram submetidas ao processo de desbaste com objetivo de obter uma superfície plana para embutimento.

Em função da necessidade de promover uma superfície condutora para análise ao MEV-EBSD, foi introduzido um material a base de grafita - CONDUCTIVE FILLER 20-8500®, BUEHLER à resina em pó CLAROFAST STRUERS $®$ na proporção 2:1,5. Após amostra embutida, foi realizado o processo de impregnação dos poros com uma mistura mais concentrada de grafita (1:2). Todos os procedimentos foram realizados na embutidora a quente - PRE30-MI®, AROTEC.

O processo de desbaste com lixa e polimento a base de pasta de diamante foi reformulado a partir da dureza resultante da mistura condutora para embutimento. A

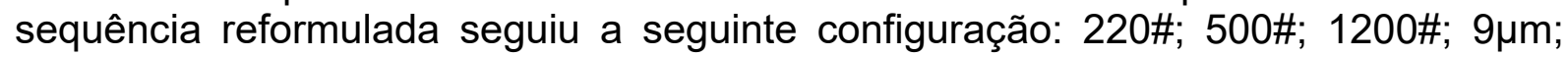
$6 \mu \mathrm{m} ; 3 \mu \mathrm{m} ; 1 \mu \mathrm{m} ; 0,25 \mu \mathrm{m} ; 0,10 \mu \mathrm{m}$, realizada em uma politriz de rotações inversas (300 x 150 RPM) - TEGRAMIM 25®, STRUERS.

A obtenção de imagens forescatter está atrelada ao refino do polimento utilizado em microscopia ótica. O polimento químico-mecânico é a etapa final do processo no qual se empregam abrasivos que, além da ação mecânica de desbaste, submetem a superfície da amostra a reações químicas em pH básico, resultando na remoção de uma fina camada do material, logo expondo uma nova superfície livre de tensões e riscos [11]. As amostras polidas mecanicamente foram submetidas ao polimento químico- mecânico por solução de sílica coloidal contendo $50 \%$ de sólidos, com tamanhos entre $20 \mathrm{~nm}-70 \mathrm{~nm}$.

\subsection{Análise microscópica}

O microscópio eletrônico de varredura utilizado durante as análises consta de um modelo JEOL JSM 5510, com resolução de 3,5nm, aceleração de voltagem de até $30 \mathrm{kV}$ e distância de trabalho mínima de $6 \mathrm{~mm}$, é equipado com um detector de elétrons retro-espalhados bem como de elétrons secundários e trabalha em condições de alto vácuo.

$O$ detector NordlysS de elétrons retroespalhados (EBSD), fabricado pela Oxford$\mathrm{HKL}$, é parte também acoplada ao Microscópio Eletrônico de Varredura (MEV). Facilmente retrátil segundo a condição de análise, é equipado com uma câmera digital CCD integrada a um chip com 12-bit (velocidade do clock de $20 \mathrm{MHz}$, resolução 1344 x 1024 pixels, binning de 8x8 com alta velocidade de leitura acima de 106 padrões de difração de elétrons por segundo, mínima distorção de imageamento). $\mathrm{O}$ equipamento de coleta digital bem como a câmera CCD acoplada são controlados pelo programa Channel 5. O detector posiciona-se a $0,1 \mathrm{~mm}$ da câmera, sendo completamente motorizado. O ângulo de captura dos padrões cristalográficos pode ser calibrado entre $15^{\circ}$ e $130^{\circ}$ sem alterações no foco, dependente apenas do sistema MEV.

As condições de contorno exigidas para a realização das análises do EBSD em conjunto com o MEV são: ângulo entre feixe e superfície da amostra de $20^{\circ}$, spot size do feixe de 70 (segundo escala de resolução do feixe de elétrons modelo JSM5510, JEOL), voltagem de $20 \mathrm{kV}$ e distância de trabalho de $32 \mathrm{~mm}$ entre a peça polar do microscópio e a superfície de interação do feixe-amostra. 
Além do programa de aquisição de dados (exposição, análise, indexação e captura), o pacote Channel 5 (Oxford-HKL) fornece programas para o tratamento desses dados. Foram utilizadas basicamente todas as ferramentas que o pacote dispõe para a análise qualitativa e quantitativa da superfície da amostra.

\section{RESULTADOS E DISCUSSÃO}

Os resultados de composição química estão listados na tabela a seguir.

Tabela 1. Composição química das pelotas analisadas

\begin{tabular}{cccccccccc}
\hline & \multicolumn{7}{c}{ Composição Química de Pelota } \\
\cline { 2 - 10 } Amostra & $\mathrm{Fe}$ calc & $\mathrm{SiO}_{2}$ & $\mathrm{Al}_{2} \mathrm{O}_{3}$ & $\mathbf{P}$ & $\mathbf{M n}$ & $\mathrm{CaO}$ & $\mathrm{MgO}$ & $\mathrm{TiO}_{2}$ & $\mathrm{C}_{\text {fixo }}$ \\
& {$[\%]$} & {$[\%]$} & {$[\%]$} & {$[\%]$} & {$[\%]$} & {$[\%]$} & {$[\%]$} & {$[\%]$} & {$[\%]$} \\
4023 & 67,87 & 1,41 & 0,38 & 0,02 & 0,04 & 0,83 & 0,04 & 0,10 & 1,00 \\
\hline
\end{tabular}

Com introdução da resina condutora, os processos de embutimento, desbaste e polimento foram reformulados com objetivo de reduzir as ocorrências de "arrancamento" e bolhas na estrutura das pastilhas. Os tempos de polimento foram elevados e a força aplicada foi reduzida. A figura a seguir ilustra a qualidade das pastilhas.
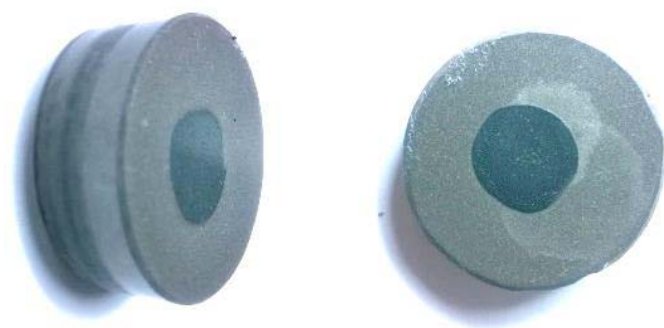

Figura 2. Pastilhas preparadas com resina condutora a base de grafita

\subsection{Mapas de fases minerais e ilustrações Forescatter}

Tabela 2. Compilação dos dados obtidos pelo tratamento estatístico dos mapas de fases gerados pelo EBSD

\begin{tabular}{|c|c|c|c|c|c|c|c|c|c|c|c|c|c|c|c|}
\hline \multirow{4}{*}{$\begin{array}{l}\text { Perfil } \\
\text { térmico }\end{array}$} & \multirow{4}{*}{ Amostra } & \multirow{3}{*}{ ccs } & \multirow{4}{*}{$\begin{array}{c}\text { Seção } \\
\text { transversal }\end{array}$} & \multirow{4}{*}{ Poros } & \multicolumn{11}{|c|}{ Fases minerais } \\
\hline & & & & & \multicolumn{3}{|c|}{ Óxidos de ferro } & \multicolumn{5}{|c|}{ Silicatos } & \multicolumn{3}{|c|}{ Ferritos } \\
\hline & & & & & Hematita & Magnetita & Wustita & Quartzo & Fayalita & Diopsidio & $\begin{array}{l}\text { Dicálcio } \\
\text { Silicato }\end{array}$ & SFCA & $\begin{array}{l}\text { Cálcio } \\
\text { Ferrita }\end{array}$ & $\begin{array}{c}\text { Dicálcio } \\
\text { Ferrita }\end{array}$ & $\begin{array}{c}\text { Magnesio- } \\
\text { ferrita }\end{array}$ \\
\hline & & [Dan/pellet] $]$ & & & $\mathrm{Fe}_{2} \mathrm{O}_{3}$ & $\mathrm{Fe}_{3} \mathrm{O}_{4}$ & $\mathrm{FeO}$ & $\mathrm{SiO}_{2}$ & 2.FeO.SiO2 & $\mathrm{CaMgSi}_{2} \mathrm{O}_{6}$ & $\mathrm{CaSiO}_{4}$ & $\mathrm{Ca}_{2,8 \mathrm{Fe}}, 7 \mathrm{Al}_{1,2} \mathrm{Si}_{0,8 \mathrm{O}_{2}}$ & $\mathrm{CaFe}_{2} \mathrm{O}_{4}$ & $\mathrm{CaF}_{2} \mathrm{O}_{5}$ & $\mathrm{MgFe}_{2} \mathrm{O}_{4}$ \\
\hline \multirow{9}{*}{ Padrão } & \multirow{4}{*}{$\begin{array}{c}4023 \\
\text { A }\end{array}$} & \multirow{4}{*}{342,00} & Casca & $28,46 \%$ & $65,12 \%$ & $0,45 \%$ & $0,08 \%$ & $0,34 \%$ & $4,21 \%$ & $0,43 \%$ & $0,02 \%$ & $0,04 \%$ & $0,17 \%$ & $0,27 \%$ & $0,41 \%$ \\
\hline & & & Mant. Int. & $34,54 \%$ & $60,12 \%$ & $0,28 \%$ & $0,06 \%$ & $0,04 \%$ & $3,80 \%$ & $0,44 \%$ & $0,02 \%$ & $0,04 \%$ & $0,17 \%$ & $0,22 \%$ & $0,27 \%$ \\
\hline & & & $\begin{array}{l}\text { Mant. Ext. } \\
\text { Núcleo }\end{array}$ & $28,00 \%$ & $67,61 \%$ & $0,13 \%$ & $0,06 \%$ & $0,01 \%$ & $\begin{array}{l}2,38 \% \\
2,99 \%\end{array}$ & $\begin{array}{l}0,56 \% \\
0,27 \%\end{array}$ & $\begin{array}{l}0,02 \% \\
0,02 \%\end{array}$ & $\begin{array}{l}0,03 \% \\
0,06 \%\end{array}$ & $\begin{array}{l}0,06 \% \\
0,25 \%\end{array}$ & $\begin{array}{l}0,27 \% \\
0,52 \%\end{array}$ & $\begin{array}{l}0,10 \% \\
0,08 \%\end{array}$ \\
\hline & & & TOTAL & $28,45 \%$ & $66,49 \%$ & $0,26 \%$ & $0,06 \%$ & $0,17 \%$ & $3,40 \%$ & $0,43 \%$ & $0,02 \%$ & $0,04 \%$ & $0,16 \%$ & $0,32 \%$ & $0,22 \%$ \\
\hline & \multirow{5}{*}{$\begin{array}{c}4023 \\
\text { B }\end{array}$} & \multirow{5}{*}{234,00} & Casca & $33,22 \%$ & $56,83 \%$ & $1,33 \%$ & $0,04 \%$ & $0,05 \%$ & $5,44 \%$ & $1,01 \%$ & $0,13 \%$ & $0,04 \%$ & $0,15 \%$ & $0,44 \%$ & $1,32 \%$ \\
\hline & & & Mant. Int. & $37,92 \%$ & $53,40 \%$ & $1,28 \%$ & $0,02 \%$ & $0,24 \%$ & $4,07 \%$ & $1,35 \%$ & $0,02 \%$ & $0,06 \%$ & $0,13 \%$ & $0,32 \%$ & $1,19 \%$ \\
\hline & & & Mant. Ext. & $40,58 \%$ & $50,38 \%$ & $1,21 \%$ & $0,01 \%$ & $0,16 \%$ & $4,43 \%$ & $1,06 \%$ & $0,01 \%$ & $0,06 \%$ & $0,22 \%$ & $0,59 \%$ & $1,29 \%$ \\
\hline & & & Núcleo & $35,00 \%$ & $56,11 \%$ & $0,95 \%$ & $0,01 \%$ & $0,21 \%$ & $5,43 \%$ & $0,76 \%$ & $0,03 \%$ & $0,04 \%$ & $0,22 \%$ & $0,31 \%$ & $0,93 \%$ \\
\hline & & & TOTAL & $36,68 \%$ & $54,18 \%$ & $1,19 \%$ & $0,02 \%$ & $0,17 \%$ & $4,84 \%$ & $1,04 \%$ & $0,05 \%$ & $0,05 \%$ & $0,18 \%$ & $0,42 \%$ & $1,18 \%$ \\
\hline \multirow{10}{*}{ Suave } & \multirow{5}{*}{$\begin{array}{c}4023 \\
C\end{array}$} & \multirow{5}{*}{319,00} & Casca & $22,84 \%$ & $72,88 \%$ & $0,36 \%$ & $0,20 \%$ & $0,03 \%$ & $2,61 \%$ & $0,20 \%$ & $0,03 \%$ & $0,04 \%$ & $0,17 \%$ & $0,25 \%$ & $0,39 \%$ \\
\hline & & & Mant. Int. & $30,04 \%$ & $62,62 \%$ & $1,39 \%$ & $0,03 \%$ & $0,05 \%$ & $3,83 \%$ & $0,31 \%$ & $0,02 \%$ & $0,04 \%$ & $0,29 \%$ & $0,22 \%$ & $1,16 \%$ \\
\hline & & & Mant. Ext. & $30,00 \%$ & $59,51 \%$ & $2,87 \%$ & $0,05 \%$ & $0,32 \%$ & $3,55 \%$ & $0,49 \%$ & $0,03 \%$ & $0,04 \%$ & $0,22 \%$ & $0,24 \%$ & $2,68 \%$ \\
\hline & & & Núcleo & $32,92 \%$ & $61,08 \%$ & $0,39 \%$ & $0,02 \%$ & $0,03 \%$ & $4,43 \%$ & $0,02 \%$ & $0,06 \%$ & $0,04 \%$ & $0,16 \%$ & $0,45 \%$ & $0,40 \%$ \\
\hline & & & TOTAL & $28,95 \%$ & $64,02 \%$ & $1,25 \%$ & $0,08 \%$ & $0,11 \%$ & $3,61 \%$ & $0,26 \%$ & $0,03 \%$ & $0,04 \%$ & $0,21 \%$ & $0,29 \%$ & $1,16 \%$ \\
\hline & \multirow{5}{*}{$\begin{array}{c}4023 \\
\text { D }\end{array}$} & \multirow{5}{*}{210,00} & Casca & $37,32 \%$ & $54,44 \%$ & $0,62 \%$ & $0,10 \%$ & $1,49 \%$ & $4,12 \%$ & $0,56 \%$ & $0,04 \%$ & $0,07 \%$ & $0,16 \%$ & $0,38 \%$ & $0,70 \%$ \\
\hline & & & Mant. Int. & $35,40 \%$ & $58,82 \%$ & $0,24 \%$ & $0,04 \%$ & $0,30 \%$ & $4,11 \%$ & $0,35 \%$ & $0,02 \%$ & $0,04 \%$ & $0,15 \%$ & $0,33 \%$ & $0,20 \%$ \\
\hline & & & Mant. Ext. & $32,03 \%$ & $61,60 \%$ & $0,23 \%$ & $0,04 \%$ & $0,70 \%$ & $3,57 \%$ & $0,87 \%$ & $0,02 \%$ & $0,07 \%$ & $0,25 \%$ & $0,36 \%$ & $0,26 \%$ \\
\hline & & & Núcleo & $26,05 \%$ & $68,92 \%$ & $0,11 \%$ & $0,05 \%$ & $0,10 \%$ & $4,02 \%$ & $0,21 \%$ & $0,01 \%$ & $0,03 \%$ & $0,12 \%$ & $0,26 \%$ & $0,12 \%$ \\
\hline & & & TOTAL & $32,70 \%$ & $60,95 \%$ & $0,30 \%$ & $0,06 \%$ & $0,65 \%$ & $3,96 \%$ & $0,50 \%$ & $0,02 \%$ & $0,05 \%$ & $0,17 \%$ & $0,33 \%$ & $0,32 \%$ \\
\hline
\end{tabular}


A amostra de pelotas verdes 4023 sofreu tratamento térmico em forno piloto adotando perfis de temperatura diferenciados, ou seja, um representando aporte térmico padrão e outro suave comparado com o praticado na planta industrial. As demais condições de contorno foram mantidas conforme o processo operacional.

Observando a formação de poros (tabela 2), as amostras de compressão baixa (figura 3, conjunto B e figura 4, conjunto D) apresentaram um percentual maior de poros em relação as amostras de compressão média (figura 3, conjunto A e figura 4, conjunto C). Segundo Lu, 1990 [12] e Meyer, 1980 [5], a combustão do carvão proporciona a formação de uma fase mineral frágil chamada Fayalita (equação 9) que, juntamente com a liberação de dióxido de carbono interferem na estrutura da pelota contribuindo diretamente para a redução da compressão. A geração de poros em excesso nas amostras $B, C$ e $D$, neste caso, pode estar relacionada diretamente a este fenômeno de formação.

A propósito, do ponto de vista de resistência física, as amostras $B, C, D$ resultaram em compressão inferior a amostra A. Visualmente (figuras 3 e 4) pode-se inferir que a formação dispersa de fases minerais mais frágeis como magnetita (equação 10) e magnésioferrita (equação 6) na matriz hematítica interfere diretamente na obtenção da propriedade física adequada para a aplicação do produto. Segundo Jhon J. et ali [2] a difusão de $\mathrm{Mg}$ +2 na matriz estabiliza a formação da magnésioferrita e, por consequência, estabiliza a temperatura de formação da magnetita. Os autores relataram influência negativa na obtenção da qualidade física com presença das fases citadas na matriz.

A combinação de fundentes como o calcário e o minério de ferro é uma das mais aventadas pela bibliografia (equação 5 e 8), de forma que, o sistema ternário cálcioferro-sílica gera componentes que são direcionados como os vetores principais para a obtenção de propriedades dos produtos metalúrgicos. Entretanto os resultados obtidos pela difração de elétrons retroespalhados realizada nas pelotas envolvidas no presente estudo endossaram a afirmação de Jhon J. et ali [2]. Os autores relataram baixa ocorrência de fases ferritas e silicatos de cálcio em pelotas com basicidade $\left(\mathrm{CaO} / \mathrm{SiO}_{2}\right)$ abaixo de 0,8 - $\mathrm{B} 2=0,59$ para as pelotas analisadas. Desta forma, concluíram que o vetor principal da resistência física para este tipo de produto é o processo de recristalização da hematita em temperaturas elevadas (acima de $1300^{\circ} \mathrm{C}$ ). Todas as amostras apresentaram percentual de cálcio ferritas reduzidos, sendo que para a amostra com resultado de compressão mais elevada, amostra A, observa-se um grau de maturação mais completo (coalescência dos grãos hematítico). 
$46^{\circ}$ Redução

$17^{\circ}$ Minério de Ferro

$4^{\circ}$ Aglomeração

ISSN 2176-3135

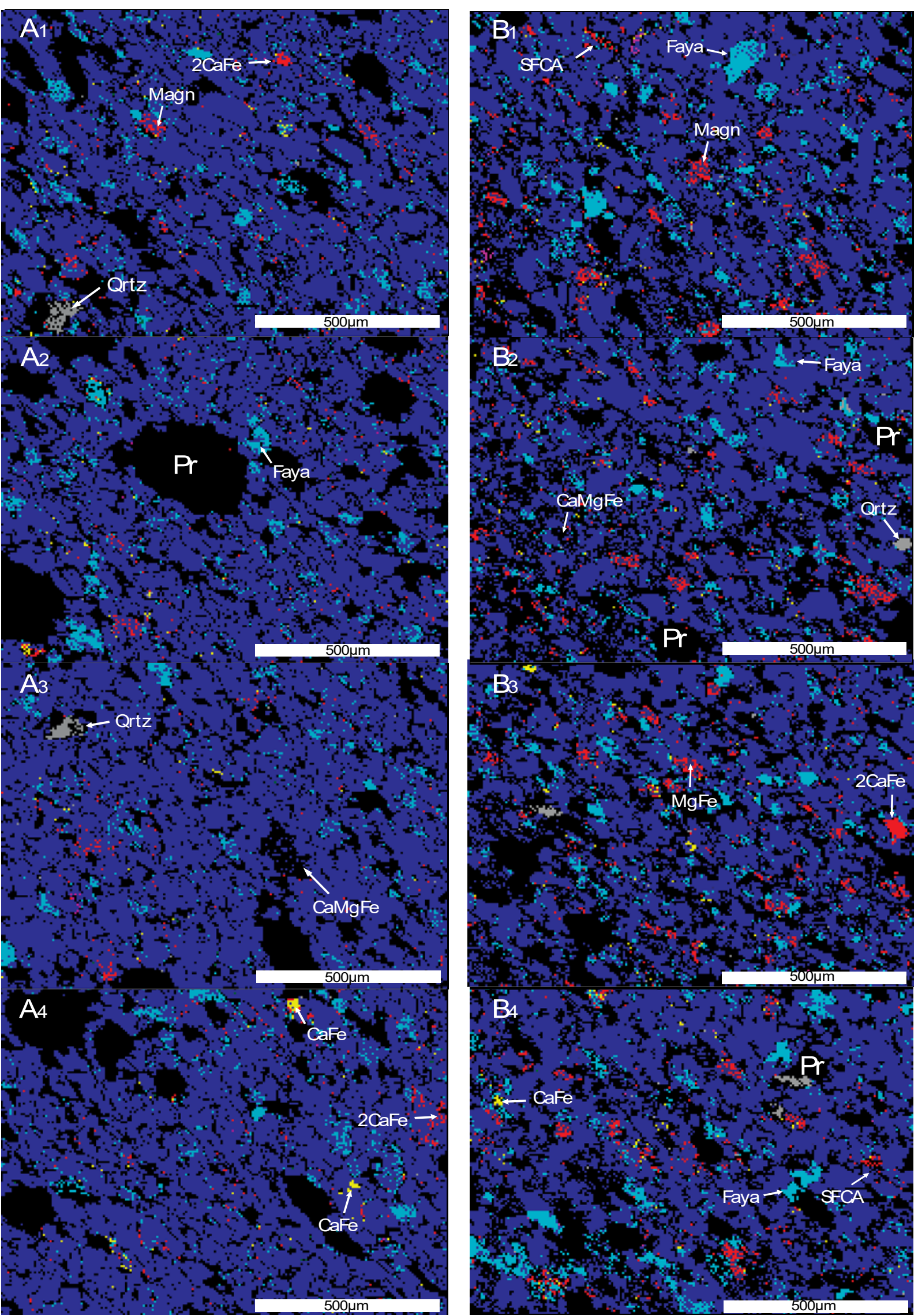

Figura 3. Amostra 4023 em perfil de temperatura padrão / amostra A - compressão média; B- compressão baixa / regiões de análises - Casca (A1 e B1); manto externo (A2 e B2); manto externo (A3 e B3); núcleo (A4 e B4) : imagens "forescatter" (田D) que ilustram as microestruturas das pelotas tratadas termicamente. Matriz de hematita (azul escuro) com presença de póros (Pr); fayalita (Faya); magnetita (Magn); diopsita (CaMgFe); quartzo (Qrtz); calcio ferrita (CaFe); dicalcio ferrita (2CaFe); magnesioferrita (MgFe) e alimínio- silicato de cálcio ferrita (SFCA) 
$46^{\circ}$ Redução

$17^{\circ}$ Minério de Ferro

$4^{\circ}$ Aglomeração
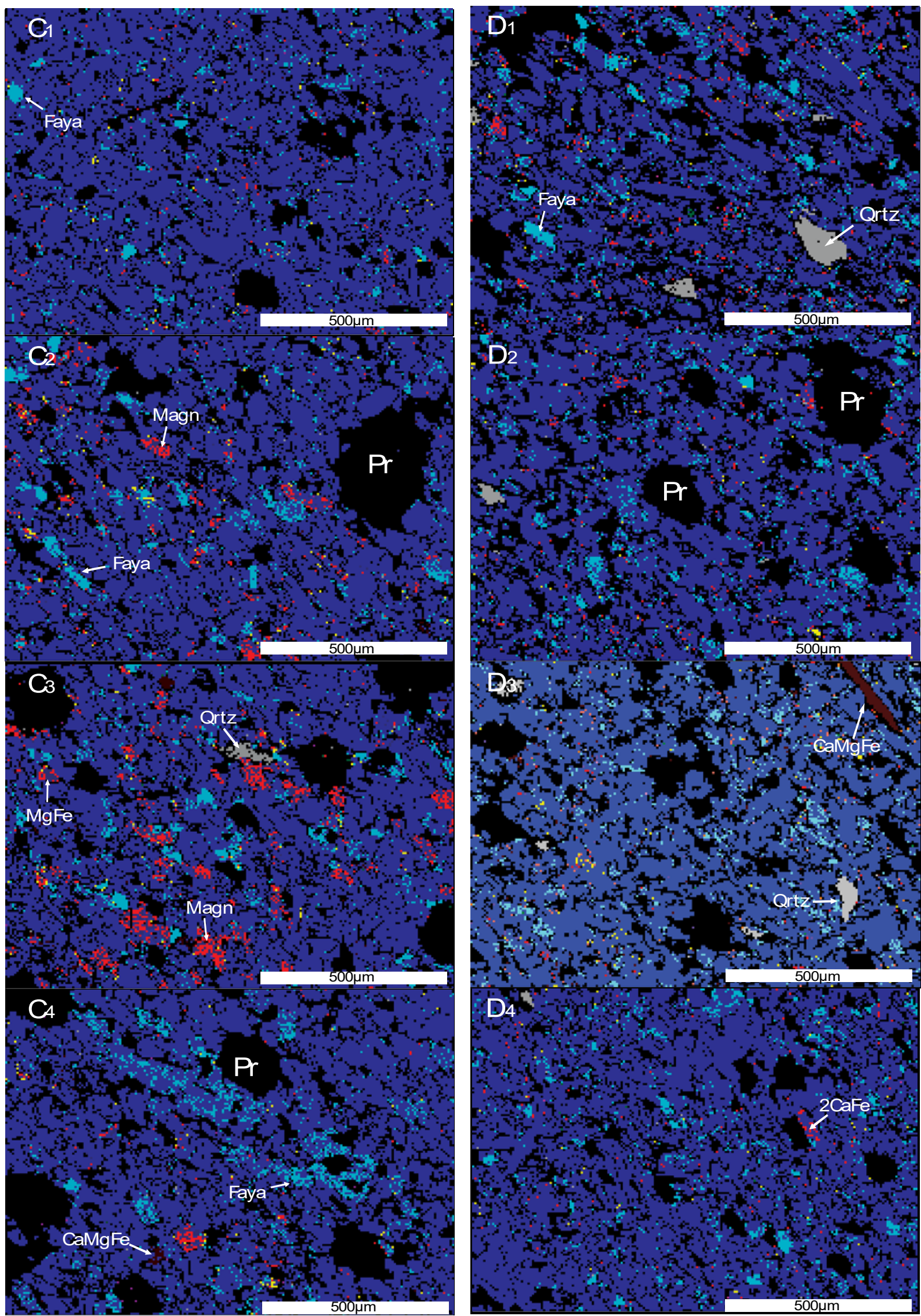

Figura 4. Amostra 4023 em perfil de temperatura suave / amostra - compressão média: D - compressão baixa/ regiões de análises - Casca (C1 e D1); manto externo (C2 e D2); manto externo (C3 e D3); núcleo (C4 e D4) : imagens"forescatter " (BSD) que ilustram as microestruturas das pelotas tratadas termicamente. Matriz de hematita (azul escuro) com presença de póros (P); fayalita (Faya); magnetita (Magn); diopsita (CaMgFe); quartzo (Qrtz); calcio ferrita (CaFe); dical cio ferrita (2CaFe); magnesioferrita (MgFe) e alimínio- silicato de cálcio ferrita (SFCA) 


\section{CONCLUSÃO}

Para a obtenção de resultados contundentes por meio de microscopia eletrônica de varredura em conjunto da técnica EBSD, a preparação de amostras torna-se o processo crucial para análises microestruturais;

O emprego da técnica de EBSD comprovou, através do mapeamento de fases minerais, a obtenção dos principais componentes formadores da microestrutura de pelotas por reações químicas aventadas pela bibliografia. Diante dos resultados gerados pelo analisador de imagens, algumas correlações com a resistência a compressão foram verificadas;

A obtenção de fases minerais como a fayalita, magnetita e magnésio-ferrita influenciam negativamente na resistência a compressão. Foi observado que quanto maior a ocorrência, menor a resistência e maior a formação de poros;

Para o produto Vale para redução direta $(B 2<0,60)$ as ocorrências de ferritos e silicatos de cálcio são reduzidos em função da baixa participação de $\mathrm{CaO}$. Deste modo, o vetor principal para a obtenção da propriedade física ideal é o grau de maturação elevado da matriz hematítica e a formação minimizada de fases minerais fragilizantes sobre o controle da composição química e aporte térmico aplicado.

A técnica desenvolvida com a aplicação da análise EBSD futuramente pode ser empregada como uma das principais fontes para entendimento e controle do comportamento metalúrgico dos processos de tratamento térmico de pelotas de minério de ferro.

\section{Agradecimentos}

Ao laboratório de microanálises MICROLAB do Departamento de Geologia - Escola de Minas, Universidade Federal de Ouro Preto, MG; planta piloto do Departamento de Pelotização e a Gerência de Pelotização Usinas 1 a 4 - Vale/Complexo deTubarão, ES.

\section{REFERÊNCIAS}

1 PANIGRAHY S.C., JENA B.C. and RIGAUD M. Characterization of Bonding and Crystalline Phases in Fluxed Pellets Using Peat Moss and Bentonite as Binders. METALLURGICAL TRANSACTIONS B. VOLUME 21B, JUNE 1990-463.

2 JOHN J. FRIEL AND EDWIN S. ERICKSON, JR. Chemistry, Microstructure, and Reduction Characteristics of Dolomite-Fluxed Magnetite Pellets. METALLURGICAL TRANSACTIONS B. VOLUME 1IB, JUNE 1980-233.

3 Fitton J.; Goldring D. 1966. Constitution of Iron Pellets in Relation to Time and Temperature of Firing. Journal of The Iron and Steel Institute, 452-459.

4 Trindade M.; Silva F. 1979. Identificação das Reações de Escorificação em Pelotas de Minério de Ferro Durante a Queima. Revista Metalurgia. ABM. Volume 35.

$5 \quad$ Meyer K. 1980. Pelletizing of Iron Ores. Wurzburg, Druckerei K. Triltseh.

6 SCHWARZER, Robert A., FIELD, David P., ADAMS, Brent L., KUMAR, Mukul \& SCHWARTZ, Adam J. Present state of eléctron backscatter diffraction and prospective developments. In: SCHWARTZ, Adam J., KUMAR, Mukul, ADAMS, Brent L. \& FIELD, David P. (Ed). Electron backscatter diffraction in materials Science. 2nd ed. New York: Kluwer Academic/Plenum Publishers, 2000. p. 1-20.

7 ENGLER,O \& RANDLE, V. Introduction to texture analysis: Macrotexture, Microtexture and Orientation Mapping. 2nd ed. Boca Raton: CRC Press, 2010. 
8 BARBOSA, P. F. Caracterização microestrutural e textural de agregados de magnetita do Quadrilátero Ferrífero. 2009. 81f. v65. n277. Dissertação (Mestrado). Departamento de Geologia, Universidade Federal de Ouro Preto, Ouro Preto.

9 ZHOU, W., APKARIAN, R., WANG, Z.L., JOY, D. Fundamentals of scanning electron microscope (SEM). In: Scanning Microscopy for Nanotechnology. 1st ED. New York: Springer, 2006. cap. 1, p. 1-39.Prior, D. J., Trimby, P. W. \& Weber, U. 1996. Orientation contrast imaging of microstructures in rocks using forescattered detectors in the scanning microscope. Mineralogical Magazine, 60:859-869

10 ZIPPERIAN, D. Colloidal silica polishing. In: Quality matters newsletter. Pace Technologies, Wheeling, IL. v. 2, issue 3, 2003.CE-41 - Map1

11 Lu W. K. 1990. Fluxed Pellets With High Coke Breeze Addition. Ironmaking Conferece Proceeding. 3-11. 